\title{
Computation of Semi-Analytical Solutions to Fuzzy Non-linear Integral Equations
}

\author{
Zia Ullah ${ }^{1}$, Aman Ullah ${ }^{1}$, Kamal Shah ${ }^{1}$, and D. Baleanu ${ }^{2}$ \\ ${ }^{1}$ University of Malakand \\ ${ }^{2}$ Cankaya Universitesi
}

May 8, 2020

\begin{abstract}
In this article, we use a fuzzy number in its parametric form and transform a non-linear fuzzy integral equations to its parametric form of the second kind as in the crisp case. The main focus is to solve the fuzzy non-linear integral equations for semi analytical solutions. The suggested treatment are presented for the solution of respective fuzzy non-linear integral equations including fuzzy non-linear Fredholm integral equation, fuzzy non-linear Volterra integral equations and fuzzy non-linear singular integral equation of Able's type kernel via an hybrid method of integral transform and decomposition technique. The proposed method is illustrated in details by solving few examples.
\end{abstract}

\section{Hosted file}

non linear fuzzy integral equation.pdf available at https://authorea.com/users/319808/ articles/449507-computation-of-semi-analytical-solutions-to-fuzzy-non-linear-integralequations 